\title{
Methylation in the TP53 promoter is associated with ischemic stroke
}

\author{
YAN WEI $^{1}$, ZHONGZHENG SUN $^{2}$, YUN WANG $^{1}$, ZHAOHONG XIE $^{1}$, \\ SHUNLIANG XU ${ }^{1}$, YINGYING XU ${ }^{1}$, XIAOYAN ZHOU ${ }^{1}$, \\ JIANZHONG BI ${ }^{1,3,4}$ and ZHENGYU ZHU ${ }^{1}$ \\ Departments of ${ }^{1}$ Neurology Medicine and ${ }^{2}$ Neurosurgery, Second Hospital of Shandong University, \\ Jinan, Shandong 250033; ${ }^{3}$ Institute of Neurology, Shandong University; \\ ${ }^{4}$ Key Laboratory of Translational Medicine on Neurological Degenerative Disease, \\ Shandong University, Jinan, Shandong 250000, P.R. China
}

Received August 4, 2018; Accepted March 6, 2019

DOI: $10.3892 / \mathrm{mmr} .2019 .10348$

\begin{abstract}
Cerebral ischemic stroke (IS) is a disease presenting high morbidity and mortality rates worldwide. Understanding of the pathogenesis underlying IS may facilitate the development of effective clinical therapeutic strategies and improve the prevention of this disease, decreasing its occurrence rate. Epigenetic alterations have recently attracted attention as possible mechanisms underlying IS. Additionally, tumor protein p53 (TP53) was identified to be involved in the pathophysiology of cerebral stroke. In the present study, the methylation status of the TP53 promoter was investigated in patients with IS and in age-matched healthy controls. The methylation status of the promoter of TP53 was significantly increased in patients with IS compared with healthy subjects. Additionally, the methylation level of the TP53 promoter was identified to be associated with carotid intima-media thickness, the degree of carotid atherosclerosis and the circulating levels of homocysteine in peripheral blood. The present findings may improve the understanding of the role of the epigenetic modifications of the TP53 promoter in IS pathogenesis.
\end{abstract}

\section{Introduction}

Cerebral ischemic stroke (IS), also known as cerebral infarction (CI), is an ischemic event caused by insufficient

Correspondence to: Dr Zhengyu Zhu, Department of Neurology Medicine, Second Hospital of Shandong University, 247 Beiyuan Street, Jinan, Shandong 250033, P.R. China

E-mail: zhuzy1133@126.com

Abbreviations: IS, cerebral ischemic stroke; CI, cerebral infarction; MSP, methylation-specific polymerase chain reaction; Hcy, homocysteine; CIMT, carotid intima-media thickness

Key words: tumor protein p53, methylation, ischemic stroke, epigenetics blood supply to brain tissues. Following an ischemic event, brain function may decrease within 60-90 sec, and cerebral ischemia may cause irreversible damage if untreated (1). Understanding of the pathogenesis of IS is required to develop multifactorial prevention strategies and novel therapeutic treatments in order to decrease the incidence and recurrence rates of IS.

Epigenetic alterations have been identified to be among the principal regulators involved in the pathogenesis of various pathological conditions, including cardiovascular diseases and cancer (2). Cytosine-phosphate-guanine $(\mathrm{CpG})$ islands in promoter regions may exhibit an abnormal methylation status in various diseases; hypermethylation may lead to repression of gene expression, whereas hypomethylation may cause chromosomal instability and loss of DNA imprinting (3). Accumulating evidence demonstrates that DNA methylation may serve a principal role in IS (4). The mRNA expression level of tumor protein p53 (TP53) was previously identified to be regulated by epigenetic mechanisms, and alterations in the methylation status of TP53 promoter may affect its expression level in pathological conditions, including cancer and atherosclerosis (5). The TP53 gene is located on the short arm of chromosome 17 (17p13.1) and it consists of 20,000 base pairs (bp). TP53 is a tumor suppressor gene that was previously identified to promote apoptosis, affect gene stability and inhibit tumor formation (6). Notably, TP53 hypermethylation may result in the silencing of TP53 (7). Although a previous study demonstrated that the circulating levels of TP53 were increased in patients with IS (8), to the best of the authors' knowledge, no studies have investigated the association between methylation of the TP53 promoter and IS.

In the present study, the methylation levels of the TP53 promoter were examined using DNA extracted from the peripheral blood of patients with IS. Methylation-specific polymerase chain reaction (MSP) and bisulfite sequencing polymerase chain reaction (BSP) analyses were used to investigate the methylation status of the promoter of TP53 in patients with IS. Additionally, the association between the methylation level of the TP53 promoter and the incidence of CI was investigated. 


\section{Materials and methods}

Subjects. In total, 78 patients with IS (female to male ratio, 19:20; age, 61.54 \pm 9.90 years) were enrolled in the present study. All patients were hospitalized in The Second Hospital of Shandong University (Jinan, China) between October 2010 and February 2011. All patients were diagnosed according to the diagnostic criteria established by The Fourth National Conference on Cerebrovascular Diseases in 1995 (9). The samples were collected within $48 \mathrm{~h}$ following IS. Transcranial magnetic resonance diffusion-weighted imaging identified acute infarction in conscious patients. Patients with tumors, connective tissue disease, severe liver kidney disease, neurological diseases and atrial fibrillation were excluded from the present study.

In the control group, 86 healthy subjects (female to male ratio 37:49; age, 62.12 \pm 7.06 years) were enrolled between October 2010 and February 2011. Healthy subjects did not exhibit evidence of IS in their medical history and no signs of CI were identified following physical examination. In a subset of healthy individuals, the diagnosis was confirmed by computed tomography and magnetic resonance imaging. Subjects exhibiting a history of transient ischemic attacks, cerebral hemorrhage, blood diseases, severe liver and kidney dysfunctions, immune system diseases and atrial fibrillation were excluded from the present study. All clinical studies were approved by The Local Ethics Committee of The Second Hospital of Shandong University and informed consent was obtained from all patients.

Blood sample collection and processing. In total, $2 \mathrm{ml}$ of blood was collected from each subject by venipuncture and was placed into serum separator tubes (BD Biosciences, San Jose, CA, USA). After 10-60 min, samples were centrifuged at $1,500 \mathrm{x} \mathrm{g}$ for $10 \mathrm{~min}$ at $4^{\circ} \mathrm{C}$. Serum was subsequently divided in $0.5 \mathrm{ml}$ aliquots and stored at $-80^{\circ} \mathrm{C}$.

DNA extraction and bisulfite modification. Genomic DNA was extracted from serum using a DNA extraction kit (cat. no. 69504; Qiagen GmbH, Hilden, Germany). A total of $2 \mu \mathrm{g}$ genomic DNA was denatured using $0.3 \mathrm{M} \mathrm{NaOH}$ for $15 \mathrm{~min}$ at $37^{\circ} \mathrm{C}$ in a final volume of $20 \mu 1$, and purified according to the manufacturer's protocol (Wizard ${ }^{\circledR}$ DNA clean-up Resin; cat. no. A7280; Promega Corporation, Madison, WI, USA). DNA samples were treated with sodium bisulfate to convert unmethylated cytosine to uracil without affecting methylated cytosine using an EpiTect Fast Bisulfite kit (Qiagen GmbH; cat. no. 59802), according to the manufacturer's protocol.

$B S P$ and MSP. MethPrimer (http://www.urogene. org/methprimer/) was used to design primers for the first $\mathrm{CpG}$ island downstream of the transcription start site of TP53 (10-12). The primers used in the present study were the following: Unmethylated forward (F), 5'-GTAGTTTGAATG TTTTTATTTTGGT-3' and unmethylated reverse (R), 5'-CCT ACTACACCCTCTACAAACA-3'; methylated F, 5'-GTAGTT TGAACGTTTTTATTTTGGC-3' and methylated R, 5'-CCT ACTACGCCCTCTACAAACG-3'. BSP primers were 5'-AAG ATTTTCGGGAGGAGAGG-3' and 5'-CCTAAATACCTA TATCAATACTAAATAACAA-3'. A hot start Taq polymerase (cat. no. 14966001; Invitrogen, Thermo Fisher Scientific, Inc., Waltham, MA, USA) was used to amplify the regions of interest by MSP and BSP. PCR thermocycling conditions were as follows: Initial denaturation at $95^{\circ} \mathrm{C}$ for $5 \mathrm{~min}, 40$ cycles of $30 \mathrm{sec}$ at $95^{\circ} \mathrm{C}, 30 \mathrm{sec}$ at $60^{\circ} \mathrm{C}$ and $45 \mathrm{sec}$ at $72^{\circ} \mathrm{C}$, and a final extension step at $72^{\circ} \mathrm{C}$ for $10 \mathrm{~min}$. PCR products were loaded on a $2 \%$ agarose gel and the bands were excised and purified using the Illustra GFX PCR DNA and Gel Band Purification kit (cat. no. 28-9034-70; GE Healthcare Life Sciences, Little Chalfont, UK). Directly sequencing PCR products may lead to inconclusive results, due to variability in the conversion efficiency and in the quantity of methylated sites. In order to obtain clear sequencing results, PCR products were cloned into plasmids prior to sequencing. Purified PCR products were ligated into pGEM-T easy plasmids (Promega Corporation) for $2 \mathrm{~h}$ on ice. DH5a Escherichia coli competent cells (cat. no. 18265017; Invitrogen; Thermo Fisher Scientific, Inc.) were transformed by heat shock and incubated overnight at $37^{\circ} \mathrm{C}$ on agar plates containing $100 \mu \mathrm{g} / \mathrm{ml}$ ampicillin (cat. no. BP021; Sigma-Aldrich; Merck KGaA, Darmstadt, Germany) (13). In total, $\geq 10$ colonies for each treatment were selected and expanded in lysogeny broth medium containing $50 \mu \mathrm{g} / \mathrm{ml}$ ampicillin. On the following day, plasmids were isolated using the PureLink plasmid miniprep kit (cat. no. K210010; Invitrogen; Thermo Fisher Scientific, Inc.). The sequences of plasmids containing the bisulfite-modified DNA were analyzed by Sanger sequencing (Laboratory Services, University of Guelph, Guelph, ON, Canada) and BiQ Analyzer 2.0 software (14) was used to analyze methylation patterns and draw diagrams. Human methylated DNA following bisulfite modification and unmethylated DNA (cat. no. 59568; Qiagen $\mathrm{GmbH}$ ) were used as controls for bisulfite conversion efficiency and accuracy, respectively.

Carotid intima-media thickness (CIMT) measurement. The CIMT of 49 patients with IS was examined by carotid color doppler ultrasonography (Philips iE33; Philips Medical Systems, Inc., Bothell, WA, USA). Two certified sonographers who were blinded to all clinical information performed carotid arterial scanning. Patients were placed in the supine position with slight hyperextension, and rotation of the neck to the contralateral side. CIMT measurements were collected at $10 \mathrm{~mm}$ intervals of the far wall of the right common carotid arteries. The CIMT was measured by manually examining the thickness of every free plaque lesion. The Crouse plaque score was used to calculate the severity of carotid artery atherosclerosis (15).

Identification of transcription factor binding site. TFSearch viewer 1.0 (Parallel Application TRC laboratory, RWCP, Japan) was used to identify the predicted transcription factor binding sites in the CpG islands of the TP53 promoter (16).

Homocysteine (Hcy) concentration detection. Hcy concentration was detected using an Hcy assay kit (cat. no. ab228559; Abcam, Cambridge, UK) according to the manufacturer's protocol.

Statistical analysis. The pathophysiological characteristics of the patients were examined using a $\chi^{2}$ test and Student's t-test. 


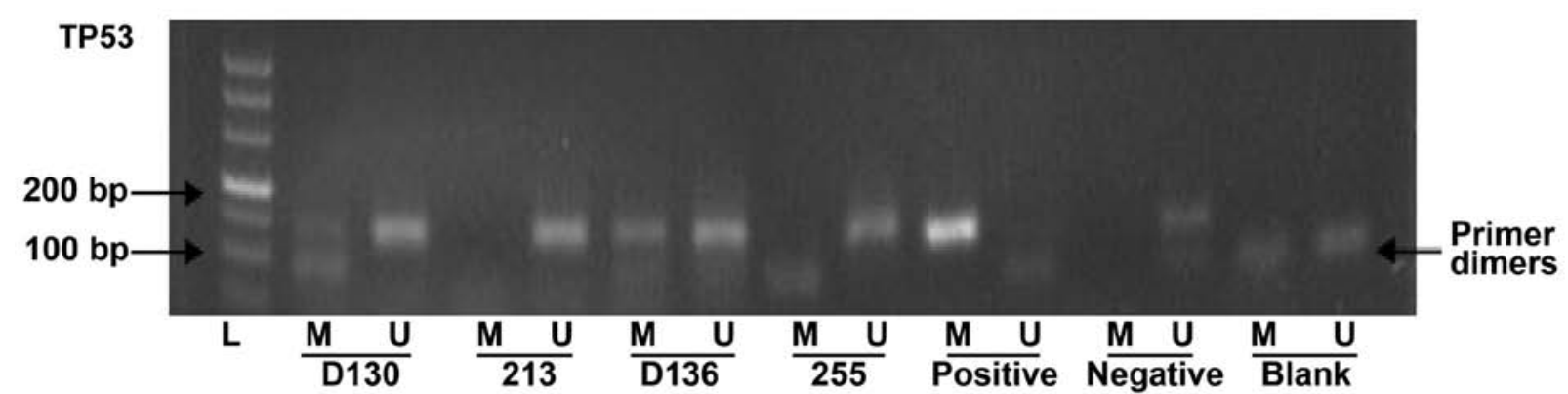

Figure 1. Electrophoresis results of methylation-specific polymerase chain reaction of TP53 promoter. Lanes D130 and D136 correspond to DNA samples from patients with ischemic stroke. Lanes 213 and 255 correspond to DNA samples from healthy subjects. Lane L, DNA marker. M, methylation-specific reaction; $\mathrm{U}$, unmethylation-specific reaction; TP53, tumor protein $\mathrm{p} 53$.

Table I. Tumor protein p53 promoter methylation in IS group and control groups.

\begin{tabular}{lcccc}
\hline Group & $\mathrm{n}$ & $\begin{array}{c}\text { Methylated } \\
\text { promoter, }\end{array}$ & $\begin{array}{c}\text { Unmethylated } \\
\text { promoter, }\end{array}$ & P-value \\
\hline IS & 78 & $25(32.1 \%)$ & $53(67.9 \%)$ & $<0.001$ \\
Control & 86 & $14(16.3 \%)$ & $72(83.7 \%)$ & \\
\hline
\end{tabular}

IS, ischemic stroke.

Associations between the methylation levels of TP53 and IS were analyzed using Student's t-test. Multivariate logistic regression analysis was used to adjust for age. SPSS software for Windows (version 17.0; SPSS, Inc., Chicago, IL, USA) was used to perform all statistical analyses. Two-tailed $\mathrm{P}<0.05$ was considered to indicate a statistically significant difference.

\section{Results}

TP53 promoter methylation in IS group and control groups. The methylation levels of TP53 promoter were determined by MSP assay (Fig 1). In total, 25 out of 78 (32.1\%) patients with IS exhibited promoter methylation. By contrast, 14 out of $86(16.3 \%)$ healthy subjects exhibited methylated CpGs. The methylation rate of the TP53 promoter in patients with IS was significantly increased compared with the control group (Table I).

Association between TP53 promoter methylation and age. Subjects were divided into two groups according to their age: i) Elderly subjects ( $\geq 60$ years); and ii) younger subjects ( $<60$ years). In total, 10 out of $37(27 \%)$ young patients and 15 out of 41 (36.6\%) elderly patients with IS exhibited methylated TP53 promoters. The rate of methylation increased with age; however, the difference was not statistically significant (Table II). In total, 5 out of 34 (14.7\%) young patients and 9 out of $52(17.3 \%)$ elderly patients in the control group exhibited methylated TP53 promoters.

Association between TP53 promoter methylation and sex. The association between the methylation level of the TP53 promoter and the sex of the subjects was investigated. The percentage of
Table II. Association between tumor protein p53 promoter methylation and age.

A, Patients with ischemic stroke

\begin{tabular}{llcc}
\hline Age, years & $\begin{array}{c}\text { Methylated } \\
\text { promoter, } n\end{array}$ & $\begin{array}{c}\text { Unmethylated } \\
\text { promoter, } n\end{array}$ & P-value \\
\hline$<60$ & $10(27.0 \%)$ & $27(73.0 \%)$ & 0.509 \\
$\geq 60$ & $15(36.6 \%)$ & $26(63.4 \%)$ & \\
\hline
\end{tabular}

B, Healthy control subjects

\begin{tabular}{llcc}
\hline Age, years & $\begin{array}{c}\text { Methylated } \\
\text { promoter, } n\end{array}$ & $\begin{array}{c}\text { Unmethylated } \\
\text { promoter, } n\end{array}$ & P-value \\
\hline$<60$ & $5(14.7 \%)$ & $29(85.3 \%)$ & 0.983 \\
$\geq 60$ & $9(17.3 \%)$ & $43(82.7 \%)$ & \\
\hline
\end{tabular}

male and female patients with IS exhibiting a methylated TP53 promoter was $35 \%(14 / 40)$ and $28.9 \%$ (11/38), respectively. The percentage of healthy male and female subjects with methylated TP53 promoters was $16.3 \%(8 / 49)$ and $16.2 \%(6 / 37)$, respectively. The difference was not statistically significant in the IS or control groups. The present results suggested that TP53 promoter methylation was not associated with sex (Table III).

Association between TP53 promoter methylation and Hcy levels in patients with IS. The circulating levels of Hcy were investigated in patients with IS exhibiting methylated and unmethylated TP53 promoters. In total, 25 patients presenting a methylated promoter of TP3 exhibited a circulating level of Hcy of 33.91 $\pm 23.81 \mu \mathrm{M}$. The concentration of Hcy in 53 patients exhibiting unmethylated TP53 promoters was $23.19 \pm 5.67 \mu \mathrm{M}$, significantly decreased compared with the methylated group. Therefore, the methylation status of TP53 was identified to be significantly associated with the circulating levels of Hcy (Table IV).

Association between TP53 promoter methylation and carotid intima-media thickness (CIMT) in patients with IS. The CIMTs 
Table III. Association between tumor protein p53 promoter methylation and sex.

A, Patients with ischemic stroke

\begin{tabular}{llcc}
\hline Sex & $\begin{array}{c}\text { Methylated } \\
\text { promoter, } n\end{array}$ & $\begin{array}{c}\text { Unmethylated } \\
\text { promoter, } n\end{array}$ & P-value \\
\hline Male & $14(35.0 \%)$ & $26(65.0 \%)$ & 0.632 \\
Female & $11(28.9 \%)$ & $27(71.1 \%)$ & \\
\hline
\end{tabular}

B, Healthy control subjects

\begin{tabular}{llll}
\hline Sex & $\begin{array}{c}\text { Methylated } \\
\text { promoter, } n\end{array}$ & $\begin{array}{c}\text { Unmethylated } \\
\text { promoter, } n\end{array}$ & P-value \\
\hline Male & $8(16.3 \%)$ & $41(83.7 \%)$ & 0.999 \\
Female & $6(16.2 \%)$ & $31(83.8 \%)$ & \\
\hline
\end{tabular}

Table IV. Association between tumor protein p53 promoter methylation and Hcy levels in patients with ischemic stroke.

\begin{tabular}{lccc}
\hline $\begin{array}{l}\text { Clinical } \\
\text { characteristic }\end{array}$ & $\begin{array}{c}\text { Methylated } \\
\text { promoter }(\mathrm{n}=25)\end{array}$ & $\begin{array}{c}\text { Unmethylated } \\
\text { promoter }(\mathrm{n}=53)\end{array}$ & P-value \\
\hline Hcy, $\mu \mathrm{M}$ & $33.91 \pm 23.81$ & $23.19 \pm 5.67$ & $0.004^{\mathrm{a}}$ \\
\hline
\end{tabular}

${ }^{\mathrm{a}} \mathrm{P}<0.01$. Hcy, homocysteine.

Table V. Association between tumor protein p53 promoter methylation and CIMT in patients with ischemic stroke.

\begin{tabular}{lccc}
\hline $\begin{array}{l}\text { Clinical } \\
\text { characteristic }\end{array}$ & $\begin{array}{c}\text { Methylated } \\
\text { promoter }(\mathrm{n}=19)\end{array}$ & $\begin{array}{c}\text { Unmethylated } \\
\text { promoter }(\mathrm{n}=30)\end{array}$ & P-value \\
\hline CIMT, mm & $1.06 \pm 0.35$ & $0.87 \pm 0.19$ & $0.018^{\mathrm{a}}$ \\
Crouse score & $4.97 \pm 4.40$ & $2.82 \pm 2.80$ & $0.041^{\mathrm{a}}$
\end{tabular}

${ }^{\mathrm{a}} \mathrm{P}<0.05$. CIMT, carotid intima-media thickness.

of 49 patients with IS were examined by carotid color doppler ultrasonography (15). The CIMT of 19 patients exhibiting methylated promoters of TP53 was $1.06 \pm 0.35 \mathrm{~mm}$. By contrast, the CIMT of patients exhibiting unmethylated promoters was $0.87 \pm 0.19 \mathrm{~mm}$. Notably, the difference was statistically significant (Table V). Additionally, the Crouse score was used to calculate the severity of carotid artery atherosclerosis (15), and a total of 19 patients in the methylated group presented a Crouse score of $4.97 \pm 4.40$. In contrast, patients with IS in the unmethylated group presented a Crouse score of 2.82 \pm 2.80 , significantly decreased compared with that of the methylated group (Table V).

BSP analysis. The methylation status of a DNA fragment containing $32 \mathrm{CpG}$ sites in the promoter region of TP53 was assessed by BSP analysis. The methylation level of patients with IS in the methylated group was confirmed to be increased. In particular, the $23 \mathrm{rd} \mathrm{CpG}$ site was methylated in $80 \%$ of patients with IS. By contrast, the 23 rd CpG site was unmethylated in the control group (Fig. 2). A bioinformatics analysis was performed to identify the predicted transcription factor binding sites in the CpG islands of the TP53 promoter using TFSearch viewer 1.0 and the 23rd $\mathrm{CpG}$ island was identified to contain a nuclear factor I (NFI) binding site.

\section{Discussion}

Epigenetic mechanisms are important physiological mediators of development and cellular functions. Epigenetic alterations are able to regulate gene expression without affecting the DNA sequence. The mechanisms underlying epigenetic alterations include DNA methylation, histone methylation, acetylation and non-coding RNA interference $(4,17,18)$. Previous studies identified that whole genome hypomethylation, epigenetic regulation of gene expression and hypermethylation of $\mathrm{CpG}$ islands are common in tumors and in pathological conditions, and the association between DNA methylation and tumor development has attracted considerable attention (19-21). An increasing number of studies have investigated epigenetic events, and DNA methylation was identified to be associated with tumors and with various diseases including atherosclerosis, diabetes, Alzheimer's disease and autoimmune diseases (22). In the present study, the methylation of TP53 promoter was investigated in patients with IS and in healthy control subjects. Additionally, the association between the methylation status of TP53 promoter and IS was investigated.

A previous study demonstrated that TP53 may serve its roles primarily by regulating the expression levels of its downstream genes involved in arresting the cell cycle, promoting cell apoptosis and maintaining gene stability (23). Additionally, a previous study demonstrated that TP53 may serve an important role in the occurrence and development of tumors, atherosclerosis and ischemic injury (24). Crumrine et al (25) observed that knockdown of TP53 expression may protect against focal ischemic damage in transgenic mice. The protein expression level of TP53 was previously identified to be upregulated following ischemic brain injury, and TP53 was able to induce apoptosis and endothelial injury, aggravating brain injury (26). Li et al (27) suggested that TP53-immunoreactive protein and TP53 mRNA expression levels were upregulated following IS, and TP53 may affect the cellular response following IS. In the present study, the methylated status of the TP53 promoter was significantly increased in patients with IS compared with the control group, suggesting that the hypermethylation of the TP53 promoter in peripheral blood may be associated with the incidence of IS and carotid atherosclerosis. The present results suggested that the alteration in the methylation status of the TP53 promoter may be important in the occurrence of carotid atherosclerosis, promoting the pathogenesis of IS. Therefore, it was hypothesized that the increased methylation level of the TP53 promoter may promote the occurrence of IS. Additionally, the increased methylation level of the TP53 promoter may be a predictive factor of IS. 
A AAGACTCCCGGGAGGAGAGGCGAACAGCGGACGCCAATTCTTTTGAAAGCACTGTGTTCCTTAGCACCGCGGGTCGCTACG GGCCTCTTGCTGTCGCGGGATTTCGGTCCACCTTCCGATTGGGCCGCCGCATCCCGGATCAGATTTCGCGGGCGACCCACGG AACCCGCGGAGCCGGGACGTGAAAGGTTAGAAGGTTTCCCGTTCCCATCAAGCCCTAGGGCTCCTCGTGGCTGCTGGGAGTT GTAGTCTGAACGCTTCTATCTTGGCGAGAAGCGCCTACGCTCCCCCTACCGAGTCCCGCGGTAATTCTTAAAGCACCTGCAC CGCCCCCCCGCCGCCTGCAGAGGGCGCAGCAGGTCTTGCACCTCTTCTGCATCTCATTCTCCAGGCTTCAGACCTGTCTCCC TCATTCAAAAAATATTTATTATCGAGCTCTTACTTGCTACCCAGCACTGATATAGGCACTCAGG

B

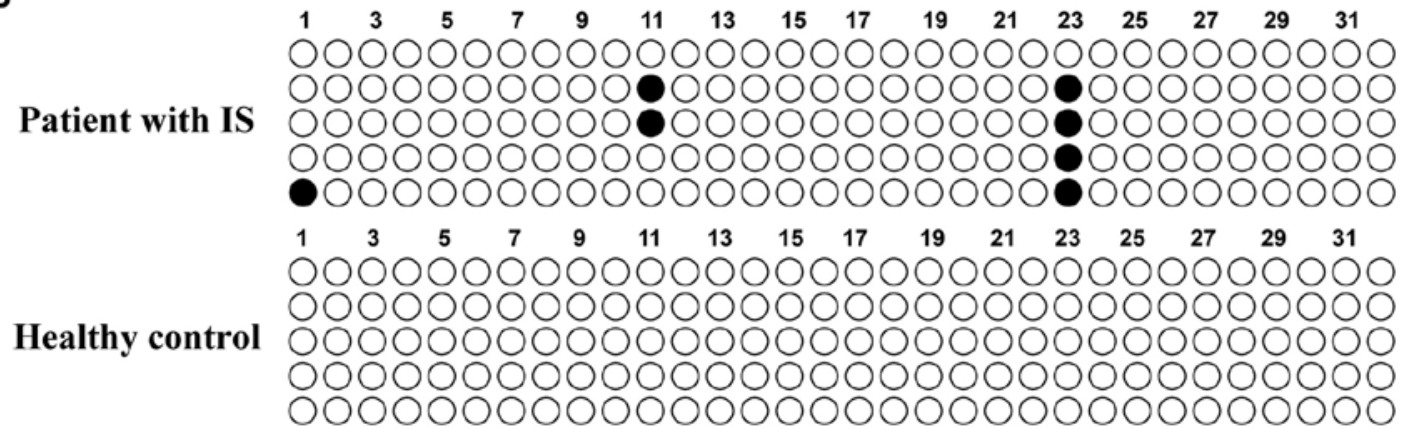

C
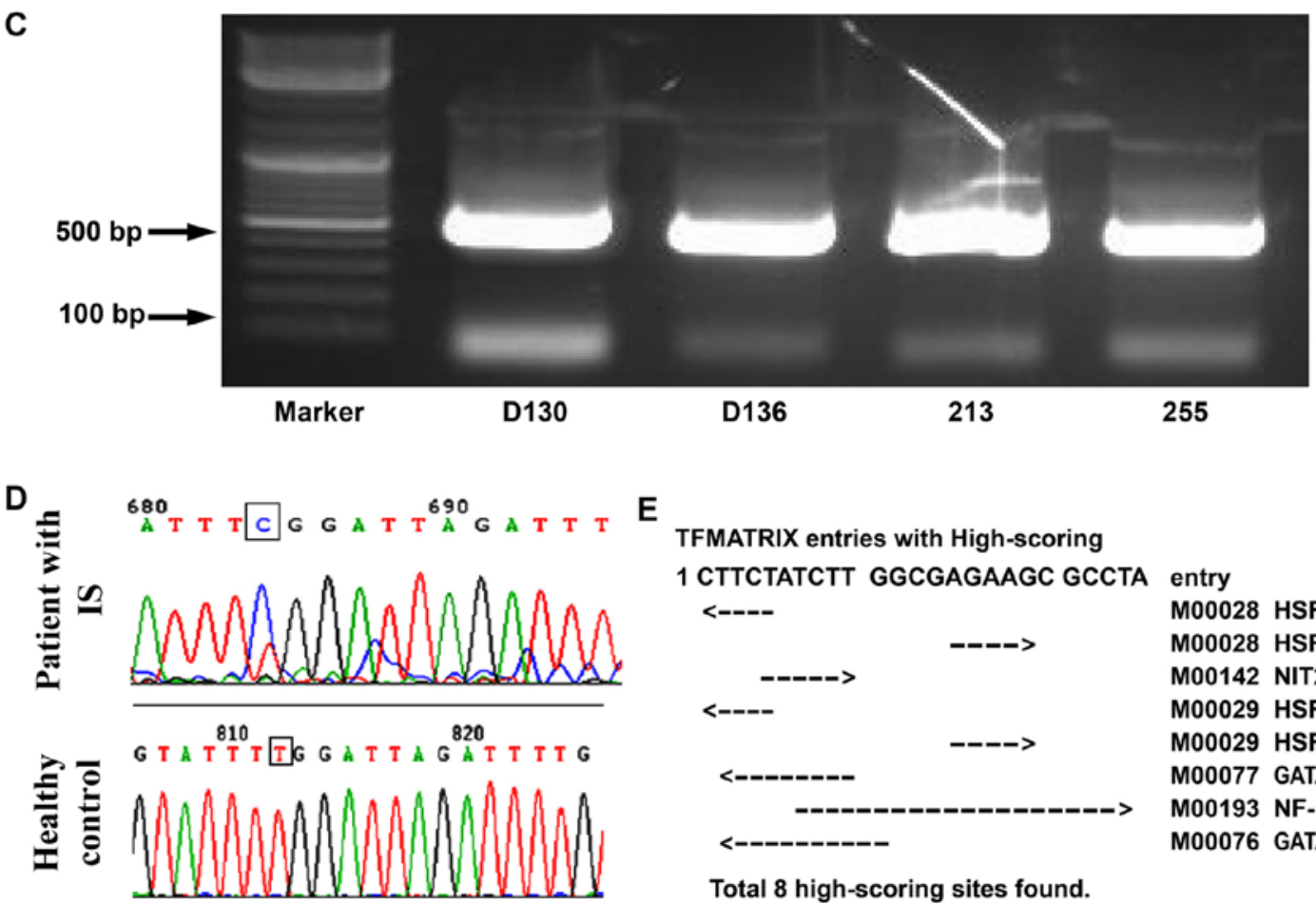

$E$

TFMATRIX entries with High-scoring

1 CTTCTATCTT GGCGAGAAGC GCCTA entry score

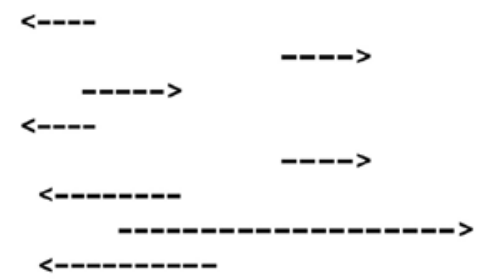

M00028 HSF 95.3

M00028 HSF 95.3

M00142 NIT2 95.0

M00029 HSF 93.7

M00029 HSF 93.7

M00077 GATA-3 86.6

M00193 NF-1 86.0

M00076 GATA-2 85.4

Total 8 high-scoring sites found.

Max score: 95.3 point $\quad$ Min score: 85.4 point

Figure 2. Results of BSP analysis of TP53 promoter. (A) BSP results for TP53 promoters and CpG sites. Underlined sequences correspond to the primers used. CpG sites are indicated in red. (B) BSP results indicated that TP53 promoter was methylated in patients with ischemic stroke. Every row corresponds to a patient or healthy control. Black circles represent methylated CpGs, and white circles represent unmethylated CpGs. (C) Electrophoresis of the amplification products used for BSP. Lanes D130 and D136 correspond to DNA samples from patients with ischemic stroke. Lanes 213 and 255 correspond to DNA samples from healthy subjects. (D) TP53 promoter sequencing in a patient with IS and a healthy control. (E) Transcription factor binding site prediction. TP53, tumor protein p53; BSP, bisulfite sequencing polymerase chain reaction; HSF, heat shock transcription factor; NIT2, nitrilase 2; NFI, nuclear factor I; CpG, Cytosine-phosphate-guanine; IS, ischemic stroke; GATA-2/3, GATA binding protein 2/3.

Carotid atherosclerosis is an important risk factor for the development of IS. Accumulating evidence demonstrates that DNA methylation may serve a role in the development of atherosclerosis (28). A previous study investigating a model of atherosclerotic lesions demonstrated that the inactivation of TP53 following promoter methylation may promote the development of atherosclerosis (29). Newman (30) demonstrated that in vivo atherosclerosis models exhibited whole genome hypomethylation. Turunen et al (31) observed that the hypermethylation of the TP53 promoter was associated with the development of atherosclerosis. Therefore, in the present study, it was hypothesized that the expression level of TP53 may be involved in regulating cell proliferation in atherosclerotic plaques. The present results suggested that the TP53 promoter region was hypermethylated in patients with IS. TP53 promoter hypermethylation may decrease the expression level of TP53, promoting the occurrence of atherosclerosis, thus serving a role in the pathogenesis of IS. 
Hcy, an amino acid containing sulfur, is an intermediate product of methionine metabolism (32). A previous study demonstrated that hyperhomocysteinemia may be a risk factor for the occurrence of cardiovascular and cerebrovascular diseases (33). The present results suggested that the circulating levels of Hcy were significantly increased in patients presenting hypermethylation of the TP53 promoter. Additionally, the results suggested that lower levels of folate may increase the concentration of Hcy in samples with methylated TP53 promoter (34). BSP analysis identified $32 \mathrm{CpG}$ sites in the TP53 promoter, and MSP analysis identified that these CpG sites were methylated in $32.1 \%$ (25/78) of patients with IS and in $16.3 \%$ (14/86) of control subjects. In particular, the $23 \mathrm{rd} \mathrm{CpG}$ island in the TP53 promoter was methylated in $80 \%$ of the samples in the methylated group. The present results suggested that hypermethylation of the TP53 promoter may be associated with the occurrence of IS. Moreover, a bioinformatics analysis identified that the $23 \mathrm{rd} \mathrm{CpG}$ island contained a nuclear NFI binding site. Therefore, methylation of this $\mathrm{CpG}$ island may affect the binding between NFI and the promoter of TP53, thus decreasing the expression level of TP53.

In conclusion, the present study suggested that methylation of TP53 promoter was significantly increased in patients with IS compared with healthy subjects. Additionally, the methylation level of the TP53 promoter was identified to be associated with the degree of carotid atherosclerosis and the Hcy concentration in peripheral blood. However, the protein expression level of TP53 in patients with IS requires further investigation. Additionally, further studies are required to investigate the protein expression level of TP53 and the methylation level of TP53 promoter in brain tissues. Collectively, the development of novel strategies aimed to alter the expression level and the activity of TP53 in patients with IS may improve the treatment and prevention of IS.

\section{Acknowledgements}

Not applicable.

\section{Funding}

The present work was supported by The National Natural Science Foundation of China (grant no. 81571052), Shandong Provincial Key Research and Development Program (grant no. 2017GSF218036), The Fundamental Research Funds of Shandong University (grant no. 2016JC022) and The Youth Foundation of The Second Hospital of Shandong University (grant no. Y2015010021).

\section{Availability of data and materials}

All data generated or analyzed during this study are included in this published article.

\section{Authors' contributions}

YWe and ZZ conceived and designed experiments. YWe, ZS, $\mathrm{ZX}$ and $\mathrm{YWa}$ performed experiments. ZX and SX provided new tools and reagents. YWe, YX, JB, SX and XZ analyzed data. YWe, JB and ZZ wrote and made manuscript revisions. All authors read and approved the final manuscript.

\section{Ethics approval and consent to participate}

All clinical studies were approved by The Local Ethics Committee of The Second Hospital of Shandong University (Jinan, China) and informed consent was obtained from all patients.

\section{Patient consent for publication}

Not applicable.

\section{Competing interests}

The authors declare that they have no competing interests.

\section{References}

1. Deb P, Sharma S and Hassan KM: Pathophysiologic mechanisms of acute ischemic stroke: An overview with emphasis on therapeutic significance beyond thrombolysis. Pathophysiology 17: 197-218, 2010.

2. Fattahi S, Pilehchian Langroudi $M$ and Akhavan-Niaki $\mathrm{H}$ : Hedgehog signaling pathway: Epigenetic regulation and role in disease and cancer development. J Cell Physiol 233: 5726-5735, 2018.

3. Matsubara N: Epigenetic regulation and colorectal cancer. Dis Colon Rectum 55: 96-104, 2012.

4. Krupinski J, Carrera C, Muino E, Torres N, Al-Baradie R, Cullell $\mathrm{N}$ and Fernandez-Cadenas I: DNA methylation in stroke. update of latest advances. Comput Struct Biotechnol J 16: 1-5, 2018.

5. Hsue SS, Wang WC, Chen YK and Lin LM: Expression of inhibitors of apoptosis family protein in 7,12-dimethylbenz[a] anthracene-induced hamster buccal-pouch squamous-cell carcinogenesis is associated with mutant p53 accumulation and epigenetic changes. Int J Exp Pathol 89: 309-320, 2008.

6. Bykov VJN, Eriksson SE, Bianchi J and Wiman KG: Targeting mutant p53 for efficient cancer therapy. Nat Rev Cancer 18: 89-102, 2018.

7. Mayr U, Mayr M, Li C, Wernig F, Dietrich H, Hu Y and Xu Q: Loss of p53 accelerates neointimal lesions of vein bypass grafts in mice. Circ Res 90: 197-204, 2002.

8. Mendrysa SM, Ghassemifar S and Malek R: p53 in the CNS: Perspectives on development, stem cells, and cancer. Genes Cancer 2: 431-442, 2011.

9. Wang X: Diagnostic essentials of various cerebrovascular diseases. Chin J Neurol 29: 379-381, 1996.

10. Li LC and Dahiya R: MethPrimer: Designing primers for methylation PCRs. Bioinformatics 18: 1427-1431, 2002.

11. Kang JH, Kim SJ, Noh DY, Park IA, Choe KJ, Yoo OJ and Kang HS: Methylation in the p53 promoter is a supplementary route to breast carcinogenesis: Correlation between $\mathrm{CpG}$ methylation in the $\mathrm{p} 53$ promoter and the mutation of the $\mathrm{p} 53$ gene in the progression from ductal carcinoma in situ to invasive ductal carcinoma. Lab Invest 81: 573-579, 2001.

12. Zhou Y, Zheng X, Lu J, Chen W, Li X and Zhao L: Ginsenoside 20(S)-Rg3 inhibits the warburg effect via modulating DNMT3A/ MiR-532-3p/HK2 pathway in ovarian cancer cells. Cell Physiol Biochem 45: 2548-2559, 2018.

13. Cohen SN, Chang AC and Hsu L: Nonchromosomal antibiotic resistance in bacteria: Genetic transformation of escherichia coli by R-factor DNA. Proc Natl Acad Sci USA 69: 2110-2114, 1972.

14. Bock C, Reither S, Mikeska T,Paulsen M, Walter J and LengauerT: BiQ analyzer: Visualization and quality control for DNA methylation data from bisulfite sequencing. Bioinformatics 21: 4067-4068, 2005.

15. Kim GH, Youn HJ, Choi YS, Jung HO, Chung WS and Kim CM: Carotid artery evaluation and coronary calcium score: Which is better for the diagnosis and prevention of atherosclerotic cardiovascular disease? Int J Clin Exp Med 8: 18591-18600, 2015. 
16. Lee $\mathrm{C}$ and Huang $\mathrm{CH}$ : Searching for transcription factor binding sites in vector spaces. BMC Bioinformatics 13: 215 , 2012.

17. Aslani S, Sobhani S, Gharibdoost F, Jamshidi A and Mahmoudi M Epigenetics and pathogenesis of systemic sclerosis; the ins and outs. Hum Immunol 79: 178-187, 2018.

18. Masser DR, Hadad N, Porter H, Stout MB, Unnikrishnan A, Stanford DR and Freeman WM: Analysis of DNA modifications in aging research. Geroscience 40: 11-29, 2018.

19. Lyu H, Huang J, He Z and Liu B: Epigenetic mechanism of survivin dysregulation in human cancer. Sci China Life Sci 61: 808-814, 2018.

20. El Bairi K, Tariq K, Himri I, Jaafari A, Smaili W, Kandhro AH, Gouri A and Ghazi B: Decoding colorectal cancer epigenomics. Cancer Genet 220: 49-76, 2018.

21. James E and Jenkins TG: Epigenetics, infertility, and cancer: Future directions. Fertil Steril 109: 27-32, 2018.

22. Mayer W, Niveleau A, Walter J, Fundele R and Haaf T: Demethylation of the zygotic paternal genome. Nature 403 : 501-502, 2000

23. Hong LZ, Zhao XY and Zhang HL: p53-mediated neuronal cell death in ischemic brain injury. Neurosci Bull 26: 232-240, 2010.

24. Crow MT: Revisiting p53 and its effectors in ischemic heart injury. Cardiovasc Res 70: 401-403, 2006.

25. Crumrine RC, Thomas AL and Morgan PF: Attenuation of $\mathrm{p} 53$ expression protects against focal ischemic damage in transgenic mice. J Cereb Blood Flow Metab 14: 887-891, 1994.
26. Li J, Chen G, Gao X, Shen C, Zhou P, Wu X, Che X and Xie R: p53 participates in the protective effects of ischemic post-conditioning against OGD-reperfusion injury in primary cultured spinal cord neurons. Neurosci Lett 638: 129-134, 2017.

27. Li Y, Chopp M, Zhang ZG, Zaloga C, Niewenhuis L and Gautam S: p53-immunoreactive protein and p53 mRNA expression after transient middle cerebral artery occlusion in rats. Stroke 25: 849-856, 1994

28. Hai Z and Zuo W: Aberrant DNA methylation in the pathogenesis of atherosclerosis. Clin Chim Acta 456: 69-74, 2016.

29. Hiltunen MO, Turunen MP, Hakkinen TP, Rutanen J, Hedman M, Mäkinen K, Turunen AM, Aalto-Setälä K and Ylä-Herttuala S: DNA hypomethylation and methyltransferase expression in atherosclerotic lesions. Vasc Med 7: 5-11, 2002.

30. Newman PE: Can reduced folic acid and vitamin B12 levels cause deficient DNA methylation producing mutations which initiate atherosclerosis? Med Hypotheses 53: 421-424, 1999.

31. Turunen MP, Aavik E and Yla-Herttuala S: Epigenetics and atherosclerosis. Biochim Biophys Acta 1790: 886-891, 2009.

32. Durand P, Prost M, Loreau N, Lussier-Cacan S and Blache D Impaired homocysteine metabolism and atherothrombotic disease. Lab Invest 81: 645-672, 2001.

33. Mok V and Kim JS: Prevention and management of cerebral small vessel disease. J Stroke 17: 111-122, 2015.

34. Joseph J and Loscalzo J: Methoxistasis: Integrating the roles of homocysteine and folic acid in cardiovascular pathobiology. Nutrients 5: 3235-3256, 2013. 